\title{
Regularization of Hyperfunctions
}

\author{
Ricardo Estrada
}

\begin{abstract}
We show that there are no continuous regularization procedures for the extension of hyperfunctions. We also show that there is a continuous projection operator from the space of hyperfunctions with support in a given compact set onto the subspace of hyperfunctions with support on a given closed subset if and only if the subset is a countable intersection of sets that are closed and open.
\end{abstract}

Keywords. Hyperfunctions, regularizations

Mathematics Subject Classification (2000). Primary 46F15, secondary 46F12

\section{Introduction}

One of the key characteristics of Sato's hyperfunctions [21, 22] is the fact that they form a flabby sheaf $[14,19]$. This means that if $U$ is an open set and $S$ is a locally closed set in $\mathbb{R}^{n}$ with $U \subset S$, then any hyperfunction $f$ defined on $U$ admits an extension $\widetilde{f}$, defined on $S$. Alternatively, the canonical projection

$$
\pi: \mathfrak{B}(S) \longrightarrow \mathfrak{B}(U)
$$

where $\mathfrak{B}(\mathcal{X})$ denotes the space of hyperfunctions defined on $\mathcal{X}$, is surjective. We say that $\tilde{f}$ is a regularization of $f$.

The regularization of generalized functions is a very important subject, not only from the mathematical point of view, but also from the mathematical physics perspective. Indeed, the problem of renormalization in quantum field theory is nothing but a problem of regularization of generalized functions $[2,23]$. Actually, the normalized coupling constants are not determined by the theory, but must be fixed by experiment, and correspond exactly to undetermined constants in regularized generalized functions.

There are several methods of regularization of generalized functions $[9,11]$ such as principal values, analytic continuation, Hadamard finite parts, or methods based on distributional continuity. For hyperfunctions one uses function

R. Estrada: Department of Mathematics, Louisiana State University, Baton Rouge, LA 70803, U.S.A; restrada@math.lsu.edu 
theoretical tools, such as the Mittag-Leffer theorem [14, 19]. Moreover, in some cases there is a controversy as to which is the best method of regularization $[9,12]$. Whether any of the regularization methods can be established as "correct" is of physical interest, because it addresses the philosophical question of whether the "infinities" in quantum field theory represent genuine physical ambiguities, or merely arise from a poor formulation of the technical mathematical problems that appear in the theory [23].

It was proved recently [7] that for distributions it is not possible to define a continuous regularization procedure that can be applied to all distributions that admit regularizations. Naturally, distributions are not a flabby sheaf and thus not all distributions admit regularizations, but this result applies to the space of extendable distributions as introduced by Orton [20]; extendable distributions were characterized in [6], and they correspond to the distributions obtained by da Silva's construction [4].

In the present article we show that the corresponding result also holds for hyperfunctions. In other words, there is no continuous extension operator

$$
R: \mathfrak{B}(a, b] \longrightarrow \mathfrak{B}[a, b]
$$

such that

$$
\pi R g=g, \quad g \in \mathfrak{B}(a, b],
$$

where $\pi: \mathfrak{B}[a, b] \longrightarrow \mathfrak{B}(a, b]$ is the restriction. The method of proof is very different from that of [7], however, since the space of distributions concentrated at one point is a $(\mathcal{D} \mathcal{F})$ space, an inductive limit of finite dimensional spaces, in fact, while the space of hyperfunctions concentrated at a point is an $(\mathcal{F})$ space.

A related question is the possibility of restriction of generalized functions to a closed set. This is a fundamental question in the study of integral equations in spaces of distributions $[10,20]$; recently it was a key component in the study of multiplicative regularizators [8], and it is expected to be play an important role in the study of hypersingular integral equations $[3,15,18]$. In this article we prove a necessary and sufficient condition for the existence of continuous restriction operators for spaces of hyperfunctions on closed sets. Indeed, we demonstrate that if $H$ and $K$ are compact sets, with $H \subseteq K$, then there exists a continuous projection operator $P: \mathfrak{B}(K) \longrightarrow \mathfrak{B}(H)$, with $P f=f$ for $f \in \mathfrak{B}(H)$, if and only if $H=\bigcap_{n=1}^{\infty} H_{n}$ where $H_{n}$ is open and closed in $K$. For spaces of distributions [7] such a projection operator exists if and only if $H$ is open in $K$. It is interesting to see how these results show how some topological conditions are related to the behavior of certain operators, a situation that is also encountered with geometric conditions [5]. It would be interesting to study the existence of restriction operators in the case when $H$ and $K$ are unbounded in the spaces of asymptotic and tempered distributions studied by Schmidt [24]. 
The plan of the article is as follows. Section 2 presents the basic ideas about hyperfunctions needed in the rest of the paper. In Section 3 we prove the nonexistence of regularization operators in spaces of hyperfunctions. Section 4 contains the study of restriction operators into spaces of hyperfunctions over a closed set.

\section{Notation and preliminaries}

In this section we state some basic facts about hyperfunctions and about the notation used in this article. For details see [14, 19, 26].

Let $U$ be an open set in $\mathbb{C}$. We denote by $\mathfrak{O}(U)$ the space of analytic functions defined on $U$. The topology of $\mathfrak{O}(U)$ is that of uniform convergence on compact subsets of $U$, i.e., the topology generated by the family of seminorms

$$
\|f\|_{K}=\max \{|f(z)|: z \in K\},
$$

for $K$ a compact subset of $U$. Since we can find a sequence of compact subsets of $U,\left\{K_{n}\right\}_{n=1}^{\infty}$ with $K_{n} \subset \operatorname{int}\left(K_{n+1}\right), \bigcup_{n=1}^{\infty} K_{n}=U$, it follows that $\mathfrak{O}(U)$ is a Fréchet space, actually a strict projective limit of Banach spaces.

A subset $S$ of a topological space $X$ is called locally closed if each $x \in S$ has a neighborhood in $X, V_{x}$, such that $S \cap V_{x}$ is closed in $V_{x}$. It can be shown that $S$ is locally closed in $X$ if and only if there exist an open set $U$ and a closed set $F$ such that $S=U \cap F$.

If $S$ is locally closed in $X$, we say that $U$ is an open neighborhood of $S$ if $U$ is open in $X$ and $S$ is closed in $U$. We denote the set of open neighborhoods of $S$ as $\mathrm{N}(S)$.

If $S$ is locally closed in $\mathbb{C}$, then $\mathfrak{O}(S)$ is the space of germs of analytic functions defined on $S$. That is, a function $f$ defined on $S$ belongs to $\mathfrak{O}(S)$ if and only if there exists $U \in \mathrm{N}(S)$ and an analytic function $\tilde{f} \in \mathfrak{O}(U)$ such that $\pi_{S}^{U}(\widetilde{f})=f$, where $\pi_{S}^{U}$ is the restriction operator from $U$ to $S$. The system of topological vector spaces $\{\mathfrak{O}(U)\}_{U \in \mathrm{N}(S)}$ with operators $\pi_{V}^{U}: \mathfrak{O}(U) \longrightarrow \mathfrak{O}(V)$ for $U \supseteq V$ is actually a directed system and thus we can give $\mathfrak{O}(S)$ the inductive limit topology. When $K$ is compact, then $\mathfrak{O}(K)$ is a strict limit of Banach spaces. If $S \subseteq \mathbb{R}$ is open, then $\mathfrak{O}(S)$ is the space of real analytic functions on $S$, while if $S \subseteq \mathbb{R}$ is locally closed, then $\mathfrak{O}(S)$ is the space of germs of real analytic functions on $S$.

If $S \subseteq \mathbb{C}$ is locally closed, then the dual space $\mathfrak{O}^{\prime}(S)$ is called the space of analytic functionals on $S$. When $K \subseteq \mathbb{R}$ is compact, then $\mathfrak{O}^{\prime}(K)$ is isomorphic to the space $\mathfrak{B}(K)$ of hyperfunctions defined on $K$, as we now explain.

If $U$ is open in $\mathbb{C}$ and $S$ is closed in $U$, then we define the space $H_{S}^{1}(U ; \mathfrak{O})$, the first cohomology group of $U$ with support in $S$ and with coefficients in the sheaf $\mathfrak{O}$, as the quotient

$$
H_{S}^{1}(U ; \mathfrak{O})=\mathfrak{O}(U \backslash S) / \pi_{U \backslash S}^{U}(\mathfrak{O}(U)),
$$


where $\pi_{U \backslash S}^{U}$ is the canonical restriction. If $U \subset V$, then the canonical homomorphism from $H_{S}^{1}(V ; \mathfrak{O})$ to $H_{S}^{1}(U ; \mathfrak{O})$ is an isomorphism as follows from the Mittag-Leffler theorem. Thus we can define $H^{1}[S ; \mathfrak{O}]$, as the inductive limit of the spaces $H_{S}^{1}(U ; \mathfrak{O})$ as $U \searrow S$. In this case, the space $H^{1}[S ; \mathfrak{O}]$ is actually isomorphic to $H_{S}^{1}(U ; \mathfrak{O})$ for any $U \in \mathbf{N}(S)$.

If $S \subseteq \mathbb{R}$ is locally closed, then the space of hyperfunctions defined on $S$ is $\mathfrak{B}(S)=H^{1}[S ; \mathfrak{O}]$. If $U \in \mathrm{N}(S)$ and $F \in \mathfrak{O}(U \backslash S)$, then the jump of $F$ across $S$,

$$
[F]=F(x+i 0)-F(x-i 0),
$$

is a hyperfunction on $S$, and actually, all hyperfunctions over $S$ have this form. Observe that this is true for all neighborhoods $U \in \mathrm{N}(S)$. In the case when $S=\mathbb{R}$ or when $S=K$, a compact subset of $\mathbb{R}$, then we can take $U=\mathbb{C}$. Thus

$$
\mathfrak{B}(\mathbb{R})=\mathfrak{O}(\mathbb{C} \backslash \mathbb{R}) / \mathfrak{O}(\mathbb{C})
$$

and

$$
\mathfrak{B}(K)=\mathfrak{O}(\mathbb{C} \backslash K) / \mathfrak{O}(\mathbb{C})
$$

If $f \in \mathfrak{B}(K)$, then of all the possible representatives in the space $\mathfrak{O}(\mathbb{C} \backslash K)$, i.e., of all the analytic functions $F \in \mathfrak{O}(\mathbb{C} \backslash K)$ with $[F]=f$, there is exactly one that satisfies

$$
\lim _{z \rightarrow \infty} F(z)=0
$$

This representative of $f$ is called the Cauchy or analytic representation of $f$, and is denoted as

$$
F(z)=\mathcal{C}\{f(x) ; z\}
$$

The operator $\mathcal{C}$ is an isomorphism of $\mathfrak{B}(K)$ onto the subspace $\mathfrak{O}_{0}(\mathbb{C} \backslash K)$ of $\mathfrak{O}(\mathbb{C} \backslash K)$ formed by those analytic functions that satisfy $(9)$. The operator $\mathcal{C}$ can also be defined as an isomorphism of the space of analytic functionals $\mathfrak{O}^{\prime}(K)$ onto $\mathfrak{O}_{0}(\mathbb{C} \backslash K)$ when $K$ is a compact subset of $\mathbb{C}$ that perhaps is not contained in $\mathbb{R}$. It can be shown that the Cauchy representation is given as

$$
\mathcal{C}\{f(\omega) ; z\}=\frac{1}{2 \pi i}\left\langle f(\omega), \frac{1}{\omega-z}\right\rangle .
$$

The power series expansion of the Cauchy representation at infinity takes the form

$$
\mathcal{C}\{f(x) ; z\}=-\frac{1}{2 \pi i} \sum_{n=0}^{\infty} \frac{\mu_{n}(f)}{z^{n+1}}, \quad|z|>\rho,
$$

where

$$
\mu_{n}(f)=\left\langle f(\omega), \omega^{n}\right\rangle,
$$

are the moments of $f$ and where $\rho=\max \{|z|: z \in K\}$. 
If $K$ is a compact subset of $\mathbb{R}$, then the topology of the space of hyperfunctions $\mathfrak{B}(K)$ is given in one of two equivalent ways. First, since $\mathfrak{B}(K)=\mathfrak{O}^{\prime}(K)$ one can give $\mathfrak{B}(K)$ the topology of the dual space. Alternatively, one could use $(8)$ and consider the quotient topology. In any case, $\mathcal{C}$ is a homeomorphism of the topological vector spaces $\mathfrak{B}(K)$ and $\mathfrak{O}_{0}(\mathbb{C} \backslash K)$.

If $S$ is a locally closed and bounded subset of $\mathbb{R}$, then $[13,25]$ one defines the topology of $\mathfrak{B}(S)$ as the quotient topology of $\mathfrak{B}(\bar{S}) / \mathfrak{B}(\bar{S} \backslash S)$. In particular,

$$
\mathfrak{B}(a, b] \cong \mathfrak{B}[a, b] / \mathfrak{B}\{a\}, \quad \mathfrak{B}(a, b) \cong \mathfrak{B}[a, b] / \mathfrak{B}\{a, b\}
$$

Notice that if $S_{1}$ is open in $S_{2}$, then the canonical projection of $\mathfrak{B}\left(S_{2}\right)$ onto $\mathfrak{B}\left(S_{1}\right)$ is continuous; likewise, if $K$ is compact, and $K \subset S$, then the canonical inclusion from $\mathfrak{B}(K)$ to $\mathfrak{B}(S)$ is continuous. Observe however that if $(a, b)$ is an open interval in $\mathbb{R}$ and $V$ is a region in $\mathbb{C}$ with $V \cap \mathbb{R}=(a, b)$, then $\mathfrak{B}(a, b) \cong \mathfrak{O}(V \backslash(a, b)) / \mathfrak{O}(V)$ algebraically but not topologically, since $\mathfrak{O}(V)$ is not closed but actually dense in $\mathfrak{O}(V \backslash(a, b))$.

\section{Regularization}

Let $a, b \in \mathbb{R}, a<b$. Let $\pi: \mathfrak{B}[a, b] \longrightarrow \mathfrak{B}(a, b]$ be the restriction operator. If $g \in \mathfrak{B}(a, b]$, then a regularization of $g$ is a hyperfunction $f \in \mathfrak{B}[a, b]$ such that $\pi f=g$. Since the hyperfunctions form a flabby sheaf $[14,19]$, the operator $\pi$ is surjective; this means that any $g \in \mathfrak{B}(a, b]$ admits regularizations. Nevertheless, regularizations are not unique since if $f_{0}$ is any regularization of $g$, then so is

$$
f(x)=f_{0}(x)+\sum_{n=0}^{\infty} c_{n} \delta^{(n)}(x-a),
$$

whenever

$$
\sum_{n=0}^{\infty} n !\left|c_{n}\right| r^{n}<\infty, \quad \forall r \geq 0 .
$$

Our first result shows that $f$, that is, $f_{0}$ and the constants $c_{n}$, cannot be chosen to depend continuously on $g$.

Theorem 1. There does not exist any continuous regularization operator

$$
R: \mathfrak{B}(a, b] \longrightarrow \mathfrak{B}[a, b]
$$

such that

$$
\pi R g=g, \quad g \in \mathfrak{B}(a, b]
$$

where $\pi: \mathfrak{B}[a, b] \longrightarrow \mathfrak{B}(a, b]$ is the canonical projection. 
Proof. Let us suppose that $R$ exists and find a contradiction. We may suppose without any loss of generality that $a=0$. Observe that if $f \in \mathfrak{B}[0, b]$, then

$$
R \pi f=f, \quad \text { for } x \neq 0,
$$

since $\pi(R \pi f)=\pi f$. Therefore, the operator $T$ defined as $T f=f-R \pi f$ is a continuous operator from $\mathfrak{B}[0, b]$ to $\mathfrak{B}\{0\}$. Hence we can write

$$
T\{f(t) ; x\}=\sum_{n=0}^{\infty} U_{n}(f) \delta^{(n)}(x),
$$

where the $U_{n}$ 's are continuous linear functionals on $\mathfrak{B}[0, b]$. Thus there are analytic functions $\phi_{n}$, each defined in a corresponding neighborhood of $[0, b]$, such that

$$
U_{n}(f)=\left\langle f(x), \phi_{n}(x)\right\rangle
$$

We will show that

$$
\phi_{n}(x)=\frac{(-1)^{n} x^{n}}{n !} \quad \forall n \in \mathbb{N} .
$$

But since $[0, b]$ is connected, it is enough to show that $\phi_{n}(x)$ and $(-1)^{n} x^{n} / n$ ! have the same Taylor series at $x=0$. But, if we observe that $T\left\{\delta^{(n)}(t) ; x\right\}=$ $\delta^{(n)}(x)$ for all $n \in \mathbb{N}$, it follows that $U_{n}\left(\delta^{(k)}(x)\right)=\delta_{k n}$ and consequently,

$$
\begin{aligned}
\phi_{n}^{(k)}(0) & =(-1)^{k}\left\langle\delta^{(k)}(x), \phi_{n}(x)\right\rangle \\
& =(-1)^{k} U_{n}\left(\delta^{(k)}(x)\right) \\
& =(-1)^{k} \delta_{k n} \\
& =\left.\frac{d^{k}}{d x^{k}}\left(\frac{(-1)^{n} x^{n}}{n !}\right)\right|_{x=0} .
\end{aligned}
$$

We conclude that

$$
T\{f(t) ; x\}=\sum_{n=0}^{\infty} \frac{(-1)^{n} \mu_{n}(f)}{n !} \delta^{(n)}(x)
$$

is precisely the Moment Expansion of $f$, since

$$
\mu_{n}(f)=\left\langle f(x), x^{n}\right\rangle=\int_{-\infty}^{\infty} f(x) x^{n} \mathrm{~d} x
$$

are the moments of $f$.

Our result now follows if we observe that the Moment Expansion of $f \in$ $\mathfrak{B}[0, b]$ does not converge in $\mathfrak{B}[0, b]$ unless $f \in \mathfrak{B}\{0\}$. Indeed, if (23) converges, then we may apply the Cauchy representation operator $\mathcal{C}$ to both sides of it. We readily obtain that $\mathcal{C}\{T(f) ; z\}=\mathcal{C}\{f ; z\}$ for $|z|>b$ and consequently for all $z \in \overline{\mathbb{C}} \backslash[0, b]$. But $\mathcal{C}\{T(f) ; z\}$ is analytic in $\overline{\mathbb{C}} \backslash\{0\}$, while $\mathcal{C}\{f ; z\}$ is analytic exactly in $\overline{\mathbb{C}} \backslash \operatorname{supp} f$. Hence supp $f=\{0\}$. 
The Theorem 1 applies to the regularization of hyperfunctions $g$ defined in the semiopen interval $(a, b]$. The situation at the endpoint $x=b$ is irrelevant, as is to be expected, and a corresponding result holds in the space $\mathfrak{B}(a, b)$.

Theorem 2. There does not exist any continuous regularization operator

$$
R: \mathfrak{B}(a, b) \longrightarrow \mathfrak{B}[a, b)
$$

such that

$$
\pi R g=g, \quad g \in \mathfrak{B}(a, b),
$$

where $\pi: \mathfrak{B}[a, b) \longrightarrow \mathfrak{B}(a, b)$ is the canonical projection.

Proof. Suppose $R$ is a continuous extension operator from $\mathfrak{B}(a, b)$ to $\mathfrak{B}[a, b)$. Let $c \in(a, b)$ and let $R_{1}=R \circ i$, where $i: \mathfrak{B}(a, c] \longrightarrow \mathfrak{B}(a, b)$ is the canonical injection. Since $R_{1}(f)(x)=0$ for $x>c$ whenever $f \in \mathfrak{B}(a, c]$, it follows that $R_{1}(f) \in \mathfrak{B}[a, c]$. Therefore, $R_{1}$ is a continuous regularization operator from $\mathfrak{B}(a, c]$ to $\mathfrak{B}[a, c]$. This contradicts the Theorem 1 .

Several remarks are in order.

Remark 1. The corresponding result for distributions was proved in [7]. The proof is quite different, however. Indeed, that proof is based on the fact that the space $\mathcal{A}_{a}$ of distributions with support in the one point set $\{a\}$ is a $(\mathcal{D} \mathcal{F})$ space, and hence if $T: \mathcal{X} \longrightarrow \mathcal{A}_{a}$ is any continuous operator from a Fréchet space $\mathcal{X}$ to $\mathcal{A}_{a}$, then there exists $N \in \mathbb{N}$ such that the order of $T(x)$ is less than or equal to $N$ for all $x \in \mathcal{X}$. On the other hand, the space $\mathfrak{B}\{a\}$ of hyperfunctions concentrated on the one point set $\{a\}$ is a Fréchet space, and thus the result on the boundedness of the orders of all $T(x)$ does not hold in the hyperfunction case.

Remark 2. Let $f$ be a hyperfunction with compact support. Let $K=\operatorname{supp} f$. Then the moment expansion

$$
\sum_{n=0}^{\infty} \frac{(-1)^{n} \mu_{n}(f)}{n !} \delta^{(n)}(x)
$$

cannot be considered as a hyperfunction, since as we demonstrated in the proof of the Theorem 1 the series (27) converges as a hyperfunction if and only if supp $f=\{0\}$. There are other useful interpretations of the moment expansion, as explained in [11, Chapter 6]. For our present purposes, we may proceed as follows. Let $\rho=\max \{|x|: x \in K\}$, and consider the closed disc $\overline{\mathbb{D}}_{\rho}$ with center at the origin and radius $\rho$, which contains $K$. Then using the Runge's theorem [19], there is an inclusion operator $i: \mathfrak{B}(K) \longrightarrow \mathfrak{O}^{\prime}\left(\overline{\mathbb{D}}_{\rho}\right)$. Moreover, 
the moment expansion of any analytic functional $g \in \mathfrak{O}^{\prime}\left(\overline{\mathbb{D}}_{\rho}\right)$ converges in this space to itself:

$$
g(z)=\sum_{n=0}^{\infty} \frac{(-1)^{n} \mu_{n}(g)}{n !} \delta^{(n)}(z), \quad g \in \mathfrak{O}^{\prime}\left(\overline{\mathbb{D}}_{\rho}\right)
$$

Needless to say, the sum of a moment series in $\mathfrak{O}^{\prime}\left(\overline{\mathbb{D}}_{\rho}\right)$ does not have support concentrated on $\{0\}$, in general.

Since $i$ is injective, it follows the moment expansion of $f \in \mathfrak{B}(K)$ determines $f$ uniquely, and it can thus be used in the hyperfunctional analysis of equations $[16,17]$.

These results do not hold for hyperfunctions of non-compact support, since there are non-zero functions whose moments all vanish [1]. On the other hand, the space of hyperfunctions where the moment asymptotic expansion holds was recently identified [24].

\section{Restriction to closed sets}

In [7] it was shown that if $H$ and $K$ are compact sets, with $H \subseteq K$, then there exists a continuous projection operator $P: \mathcal{E}^{\prime}[K] \longrightarrow \mathcal{E}^{\prime}[H]$, with $P f=f$ for $f \in \mathcal{E}^{\prime}[H]$, if and only if $H$ is open in $K$; here $\mathcal{E}^{\prime}[K]$ is the space of distributions with support contained in the compact set $K$. It is an interesting fact that this result does not hold for hyperfunction spaces; indeed, we shall show that there is a continuous projection operator $P: \mathfrak{B}(K) \longrightarrow \mathfrak{B}(H)$, with $P f=f$ for $f \in \mathfrak{B}(H)$, if and only if $H=\bigcap_{n=1}^{\infty} H_{n}$ where $H_{n}$ is open and closed in $K$.

We start with a result on countable intersection of closed-open sets.

Lemma 1. Let $H$ and $K$ be compact subsets of $\mathbb{R}$ with $H \subseteq K$. Then the following are equivalent:

a) There exist a sequence of closed and open subsets $H_{n}$ of $K$ such that

$$
H=\bigcap_{n=1}^{\infty} H_{n}
$$

b) $\forall a \in H, \forall \varepsilon>0$,

$$
(a, a+\varepsilon) \nsubseteq K \backslash H
$$

and

$$
(a-\varepsilon, a) \nsubseteq K \backslash H \text {. }
$$

Proof. Suppose that b) does not hold. Then there exists an $a \in H$ and an $\varepsilon>0$ such that (30) or (31) does not hold. To fix the ideas, suppose (30) does not 
hold, that is, $(a-\varepsilon, a) \subseteq K \backslash H$. Suppose now that $\left\{H_{n}\right\}_{n=1}^{\infty}$ is a sequence of closed and open subsets of $K$ with $H \subseteq H_{n}$ for all $n \in \mathbb{N}$. Then $\bigcap_{n=1}^{\infty} H_{n}$ cannot be equal to $H$ since $H_{n} \cap(a, a+\varepsilon)$ is open in $\mathbb{R}$ while $H_{n} \cap[a, a+\varepsilon]$ is closed and non-empty and this can happen if and only if $[a, a+\varepsilon] \subseteq H_{n}$.

Conversely, suppose b) holds. Write

$$
\mathbb{R} \backslash H=(-\infty, \widetilde{b}) \cup(\widetilde{a}, \infty) \cup \bigcup_{k \in F}\left(a_{k}, b_{k}\right)
$$

as a disjoint union of open intervals; here $F$ is a set with $|F| \leq \aleph_{0}$. Construct sequences $\left\{\widetilde{a}_{n}\right\}_{n=1}^{\infty},\left\{\widetilde{b}_{n}\right\}_{n=1}^{\infty},\left\{a_{n}^{k}\right\}_{n=1}^{\infty}$, and $\left\{b_{n}^{k}\right\}_{n=1}^{\infty}$ of $\mathbb{R} \backslash K$ such that $\widetilde{a}_{n} \searrow \widetilde{a}$, $\widetilde{b}_{n} \nearrow \widetilde{b}, a_{n}^{k}<b_{n}^{k}$, while $a_{n}^{k} \searrow a_{k}$, and $b_{n}^{k} \nearrow b_{k}$. Let $\left\{F_{n}\right\}_{n=1}^{\infty}$ be a sequence of finite sets with $\bigcup_{n=1}^{\infty} F_{n}=F$. Let

$$
U_{n}=\left(-\infty, \widetilde{b}_{n}\right) \cup\left(\widetilde{a}_{n}, \infty\right) \cup \bigcup_{k \in F_{n}}\left(a_{n}^{k}, b_{n}^{k}\right)
$$

Since $U_{n}$ is a finite union of open intervals whose endpoints do not belong to $K$, then $U_{n} \cap K$ is both open and closed in $K$. We just need to take $H_{n}=$ $K \cap\left(\mathbb{R} \backslash U_{n}\right)$ in order to satisfy a).

We are now ready to prove the ensuing characterization.

Theorem 3. Let $H$ and $K$ be compact subsets of $\mathbb{R}$ with $H \subseteq K$. Then the following statements are equivalent:

a) There exists a continuous operator

$$
P: \mathfrak{B}(K) \longrightarrow \mathfrak{B}(H),
$$

with

$$
P f=f, \quad \forall f \in \mathfrak{B}(H) .
$$

b) There exist a sequence of closed and open subsets $H_{n}$ of $K$ such that

$$
H=\bigcap_{n=1}^{\infty} H_{n} .
$$

Proof. Suppose a) holds but b) does not. Then using the lemma, there exists an $a \in H$ and an $\varepsilon>0$ such that $(a, a+\varepsilon] \subset K \backslash H$, or $[a-\varepsilon, a) \subset K \backslash H$. Suppose $(a, a+\varepsilon] \subset K \backslash H$. Without loss of generality, suppose $a=0$. Let $i_{1}$ be the injection of $\mathfrak{B}[0, \varepsilon]$ into $\mathfrak{B}(H), i_{2}$ the injection of $\mathfrak{B}(H)$ into $\mathfrak{O}^{\prime}\left(\mathbb{D}_{\rho}\right)$ where $\rho=\sup \{|x|: x \in H\}$, and set

$$
T=i_{2} \circ P \circ i_{1}
$$


Then using the fact that $[0, \varepsilon]$ is connected, the same argument used in the proof of the Theorem 1 yields the formula

$$
T(f)=\sum_{n=0}^{\infty} \frac{(-1)^{n} \mu_{n}(f)}{n !} \delta^{(n)}(z) ;
$$

that is, $T(f)$ is given by the moment expansion of $f$. But this implies that the Cauchy representation of $f$ and of $T(f), \mathcal{C}\{f(x) ; z\}$ and $\mathcal{C}\{T(f)(\omega) ; z\}$, coincide for $|z|>\rho$ and thus they have identical analytic continuations to $\overline{\mathbb{C}} \backslash H$. This in turn implies that $f=P\left(i_{1}(f)\right)$. However, this is impossible if $\operatorname{supp} f \subset[0, \varepsilon] \backslash H$.

On the other hand, if we assume that b) holds, then we can construct an operator $P$ as follows. Let $\pi_{k}: \mathfrak{B}(K) \longrightarrow \mathfrak{B}\left(H_{n}\right)$ be the projection operator; its existence is derived from the fact that the hyperfunctions are a flabby sheaf. Let $\rho=\sup _{x \in K}|x|$ and define

$$
P_{0}: \mathfrak{B}(K) \longrightarrow \mathfrak{O}^{\prime}\left(\overline{\mathbb{D}}_{\rho}\right)
$$

as the modified moment series

$$
P_{0}(f)=\sum_{n=0}^{\infty} \frac{(-1)^{n} \mu_{n}\left(\pi_{n}(f)\right)}{n !} \delta^{(n)}(z) .
$$

Then $P_{0}$ is a continuous operator. The analytic functional $P_{0}(f)$ corresponds to a hyperfunction of the space $\mathfrak{B}\left(H_{n}\right)$ for all $n \in \mathbb{N}$, and thus of the space $\mathfrak{B}(H)$. Hence there is a continuous operator $P: \mathfrak{B}(K) \longrightarrow \mathfrak{B}(H)$ with $i \circ P=P_{0}$, where $i: \mathfrak{B}(H) \longrightarrow \mathfrak{O}^{\prime}\left(\overline{\mathbb{D}}_{\rho}\right)$ is the injection. Finally we observe that $(35)$ follows from the fact that if $f \in \mathfrak{B}(H)$, then

$$
P_{0}(f)=\sum_{n=0}^{\infty} \frac{(-1)^{n} \mu_{n}(f)}{n !} \delta^{(n)}(z),
$$

is precisely the Moment Expansion of $f$.

Observe in particular that the theorem shows that if $H$ and $K$ are closed intervals with $H \subseteq K$, then there does not exist a restriction operator from $\mathfrak{B}(K)$ to $\mathfrak{B}(H)$. The nonexistence of continuous restrictions onto a closed interval is a very important result in the study of integral equations in spaces of hyperfunctions over finite intervals, the so-called finite transforms. Indeed, if $T: \mathfrak{B}(\mathbb{R}) \rightarrow \mathfrak{B}(\mathbb{R})$ is an operator, then we can define an associated operator $T_{1}: \mathfrak{B}[a, b] \rightarrow \mathfrak{B}(a, b)$ by setting $T_{1}=\pi T$, where $\pi: \mathfrak{B}(\mathbb{R}) \rightarrow \mathfrak{B}(a, b)$ is the canonical projection. Unfortunately, operators of this kind are not wellbehaved, specifically, they are not of the Fredholm type. In the ideal situation one would like to have an operator from $\mathfrak{B}[a, b]$ to itself, but in general that 
is not possible because of the results of this section. The study of such finite transforms in spaces of distributions by using multiplicative regularizators is given in [8]; several particular equations, as those of the Cauchy type, of the Carleman type, or with logarithmic kernels are considered in [10].

\section{References}

[1] Boas, R. P., The Stieljes moment problem for functions of bounded variation. Bull. Amer. Math. Soc. 45 (1939), 399 - 404.

[2] Bogoliubov, N. N and Shirkov, D. V., Introduction to the Theory of Quantized Fields. New York: Wiley 1959.

[3] Bose, S. K., Finite part representation of hyper-singular integral equations of accoustic scattering and radiation by open surfaces. Proc. Indian Acad. Sci. (Math. Sci.) 106 (1996), $271-280$.

[4] Campos Ferreira, J., Introduction to the Theory of Distributions. London: Longman 1997.

[5] David, G. and Semmes, S., Analysis of and on Uniformly Rectifiable Sets. Providence: Amer. Math. Soc. 1993.

[6] Estrada, R., Regularization of distributions. Int. J. Math. Math. Scs. 21 (1997), $625-636$.

[7] Estrada, R., The non-existence of regularization operators. J. Math. Anal. Appls. 226 (2003), 1 - 10.

[8] Estrada, R., Regularization of integral equations in spaces of distributions. J. Int. Eqns. Appls. 15 (2003), $241-262$.

[9] Estrada, R. and Fulling, S. A., How singular functions define distributions. J. Physics A 35 (2002), 3079 - 3089.

[10] Estrada, R. and Kanwal, R. P., Singular Integral Equations. Boston: Birkhäuser 2000.

[11] Estrada, R. and Kanwal, R. P., A Distributional Approach to Asymptotics. Theory and Applications (second edition). Boston: Birkhäuser 2002.

[12] Ford, G. W. and O'Connell, R. F., Derivative of the hyperbolic tangent. Nature 380 (1996), 113.

[13] Hörmander, L., The Analysis of Linear Partial Differential Operators I. New York: Springer 1983.

[14] Kaneko, A., Introduction to Hyperfunctions. Boston: Kluwer 1988.

[15] Kaya, A. C. and Erdogan, F., On the solution of integral equations with strong singular kernels. Quart. Appl. Math. XLV (1987), 105-122.

[16] Lumer, G., Introduction to hyperfunctions and $\delta$-expansions. In: Generalized functions, operator theory, and dynamical systems (Brussels, 1997). Chapman \& Hall/CRC Res. Notes Math. 399. Boca Raton, FL: Chapman \& Hall/CRC 1999, pp. $1-25$. 
[17] Lumer, G. and Neubrander, F., Signaux non-détectables en dimension $N$ dans des systèmes gouvernés par des équations de type parabolique. C. R. Acad. Sci. Paris Ser I 325 (1999), 731 - 736.

[18] Martin, P. A., Endpoint behaviour of solutions to hypersingular integral equations. Proc. Roy. Soc. London 432 (1991), $301-320$.

[19] Morimoto, M., Introduction to Sato's Hyperfunctions. Providence: Amer. Math. Soc. 1993.

[20] Orton, M., Distributions and singular integral equations of Carleman type. Applicable Anal. 9 (1979), 219 - 231.

[21] Sato, M., Theory of hyperfunctions I. J. Fac. Sci. Univ. Tokyo Ser. I 8 (1959), $139-193$.

[22] Sato M., Theory of hyperfunctions II. J. Fac. Sci. Univ. Tokyo Ser. I 9 (1960), $387-437$.

[23] Scharf, G., Finite Quantum Electromechanics: the causal approach (second edition). Berlin: Springer 1995.

[24] Schmidt, A. U., Asymptotic hyperfunctions, tempered hyperfunctions, and asymptotic expansions. Int. J. Math. Math. Scs. 2005 (2005), 755-788.

[25] Wakebayushi, S., Classical Microlocal Analysis in the Space of Hyperfunctions. Lect. Notes in Math. 1737. New York: Springer 2000.

[26] Yosida, K., Operational Calculus - A theory of hyperfunctions. Berlin: Springer 1984.

Received November 29, 2004 